\title{
Gas-Kinetic Finite Volume Methods
}

\author{
K. Xu, L. Martinelli, A. Jameson \\ Department of M.A.E, Princeton University, \\ Princeton N.J. 08544, USA
}

\section{Introduction}

Gas-kinetic schemes developed from the BGK model have been successfully applied to $1-\mathrm{D}$ and 2-D flows $[1,2]$. One of the advantages of the gas-kinetic approach over more conventional methods is realized when one considers the multidimensional Euler and Navier-Stokes equations. In fact, by requiring only a single scalar gas distribution function $f$, the gas-kinetic approach greatly simplifies the calculation of mass, momentum, energy densities and their fluxes.

In this paper we construct a novel 3-D method for the system of conservation laws of fluid flow. A Lax-Wendroff type scheme is developed from gas-kinetics and applied to solve time dependent problems. In the limit of infinite collision time $(\tau=\infty)$ our formulation reduces precisely to the kinetic representation of flux vector splitting for the Euler equations. Moreover, we introduce a new time-independent flux formulation which, when applied in conjunction with Jameson's multigrid time stepping scheme, yields efficient and accurate solutions of steady compressible flows.

\section{Three Dimensionäl Finite Volume Gas-Kinetic Schemes}

In the finite volume method, the discretization is accomplished by dividing the flow into a large number of small subdomains, and applying the conservation laws in the integral form

$$
\frac{d}{d t} \int_{\Omega} U d V+\int_{\partial \Omega} \mathbf{F} \cdot d \mathbf{S}=0
$$

to each subdomain $\Omega$ with boundary $\partial \Omega$. In this equation $U$ is the macroscopic state vector, defined as $U=\left(\rho, P_{x}, P_{y}, P_{z}, \epsilon\right)^{T}$, where $\rho, \vec{P}$, and $\epsilon$ are the mass, momentum, and energy densities, and $\mathbf{F}$ is the flux vector. In a gas-kinetic finite volume scheme the flux vectors across cell boundaries are constructed by computing the gas distribution function $f$. In three dimensions, we assume the BGK model as the governing equation for the distribution function $f$

$$
f_{t}+u f_{x}+v f_{y}+w f_{z}=(g-f) / \tau
$$

Here $f$ is a function of space $(x, y, z)$, time $t$, particle velocity $(u, v, w)$ and internal variable $\xi$ with $K$ degrees of freedom(i.e. $K=2$ for $\gamma=1.4$ gases). The relations 
between mass $\rho$, momentum $\vec{P}$ and energy $\epsilon$ densities with the distribution function $f$ are

$$
\left(\rho, P_{x}, P_{y}, P_{z}, \epsilon\right)^{T}=\int \psi_{\alpha} f d \Xi, \quad \alpha=1,2, \ldots 5
$$

where $\psi_{\alpha}$ is the vector of moments $\psi_{\alpha}=\left(1, u, v, w, \frac{1}{2}\left(u^{2}+v^{2}+w^{2}+\xi^{2}\right)\right)^{T}$ and $d \Xi=d u d v d w d \xi$ is the volume element in the phase space. In the BGK model the equilibrium state is described by a Maxwellian distribution

$$
g=A e^{-\lambda\left((u-U)^{2}+(v-V)^{2}+(w-W)^{2}+\xi^{2}\right)}
$$

where $U, V$ and $W$ are macroscopic gas velocities. For an equilibrium gas flow with $f=g$, the Euler equations in three dimensional space can be obtained by taking the moments of $\psi_{\alpha}$ in Eq.(1). On the other hand, to the first order of $\tau$, the Navier-Stokes equations, with a dynamic viscosity coefficient of $\nu=\tau p$ (where $p$ is the pressure), can be derived from the Chapman-Enskog expansion. Since mass, momentum and energy are conservative quantities in the process of gas evolution, $f$ and $g$ have to satisfy the conservation constraint

$$
\int(g-f) \psi_{\alpha} d \Xi=0, \quad \alpha=1,2, \ldots 5
$$

at any point in space and time.

The general solution for $f$ in Eq.(1) in three dimensions at the position of $(x, y, z)$ and $t$ is

$$
\begin{aligned}
f(x, y, z, t, u, v, w, \xi) & =\frac{1}{\tau} \int_{0}^{t} g\left(x^{\prime}, y^{\prime}, z^{\prime}, t, u, v, w, \xi\right) e^{-\left(t-t^{\prime}\right) / \tau} d t^{\prime} \\
& +e^{-t / \tau} f_{0}(x-u t, y-v t, z-w t)
\end{aligned}
$$

where $x^{\prime}=x-u\left(t-t^{\prime}\right), y^{\prime}=y-v\left(t-t^{\prime}\right), z^{\prime}=z-w\left(t-t^{\prime}\right)$ are the trajectory of a particle motion, and $f_{0}$ is the initial nonequilibrium gas distribution function $f$ at the beginning of each time step $(t=0)$. Two unknowns $g$ and $f_{0}$ must be determined in the above equation to obtain the solution $f$, so as to evaluate the fluxes across cell boundaries. We will consider the evaluation of fluxes across a boundary separating two cells in the $x$ direction and, to simplify the notation, the point for evaluating fluxes at the cell boundary will be assumed at $(x=0, y=0, z=0)$.

Generally, $f_{0}$ and $g$ can be expanded around the cell boundary as

$$
f_{0}= \begin{cases}g^{l}\left(1+a^{l} x+b^{l} y+c^{l} z\right), & x<0 \\ g^{r}\left(1+a^{r} x+b^{r} y+c^{r} z\right), & x>0\end{cases}
$$

and

$$
g=g_{0}(1+\bar{a} x+\bar{b} y+\bar{c} z+\bar{A} t)
$$

where $g^{l}, g^{r}$ and $g_{0}$ are local Maxwellian distribution functions. The dependence of $a^{l}, b^{l}, \ldots, \bar{A}$ on the particle velocity can be obtained from the Taylor expansion of a Maxwellian such as

$$
\bar{A}=\bar{A}_{1}+\bar{A}_{2} u+\bar{A}_{3} v+\bar{A}_{4} w+\bar{A}_{5}\left(u^{2}+v^{2}+w^{2}+\xi^{2}\right)
$$


In order to get all the parameters in Eq.(6) and Eq.(7) at time $t=0$, we need first interpolate the initial macroscopic variables. The interpolation is carried out by using a Symmetric LImited Positive (SLIP) technique originally developed by Jameson from Local Extremum Diminishing (LED) considerations [3]. All the coefficients in $f_{0}$ can be obtained directly. Then, $g_{0}$ in Eq.(7) at $(x=0, y=0, z=0, t=0)$ can be evaluated automatically by taking the limit of Eq. (5) as $t \rightarrow 0$ and substituting into Eq.(4) to obtain

$$
\int g_{0} \psi_{\alpha} d \Xi=\int_{u>0} \int g^{l} \psi_{\alpha} d \Xi+\int_{u<0} \int g^{r} \psi_{\alpha} d \Xi, \quad \alpha=1,2, \ldots 5
$$

The other terms of $\bar{a}, \bar{b}$ and $\bar{c}$ in Eq.(8) at $t=0$ can be computed from the new mass, momentum and energy interpolations which are continuous across the cell boundary in all three directions. Now, the only unknown term left in Eq.(8) is $\bar{A}$. This can be evaluated as follows by substituting Eq.(6) and Eq.(7) into Eq.(5), we get

$$
\begin{aligned}
f(0,0,0, t, u, v, w) & =\left(1-e^{-t / \tau}\right) g_{0}+\left(\tau\left(-1+e^{-t / \tau}\right)+t e^{-t / \tau}\right)(u \bar{a}+v \bar{b}+w \bar{c}) g_{0} \\
& +\tau\left(t / \tau-1+e^{-t / \tau}\right) \bar{A} g_{0}+e^{-t / \tau} f_{0}(-u t,-v t,-w t)
\end{aligned}
$$

with

$$
f_{0}(-u t,-v t,-w t)= \begin{cases}g^{l}\left(1-a^{l} u t-b^{l} v t-c^{l} w t\right), & u>0 \\ g^{r}\left(1-a^{r} u t-b^{r} v t-c^{r} w t\right), & u<0\end{cases}
$$

Both $f$ (Eq.(10)) and $g$ (Eq.(7)) contain $\bar{A}$. After applying the condition (4) at ( $x=0, y=0, z=0$ ) and integrating it over the whole time step $T$, such as

$$
\int_{0}^{T} \int(g-f) \psi_{\alpha} d \Xi d t=0
$$

five moment equations of $\bar{A}$ can obtained, from which the five constants in $\bar{A}$ of Eq.(8) can be uniquely determined. Finally, the time-dependent numerical fluxes at a cell boundary can be computed. These fluxes satisfy the consistency condition $\mathcal{F}(U, U)=\mathcal{F}(U)$ for a homogeneous uniform flow, where $\mathcal{F}(U)$ is identical to the corresponding Euler fluxes in the $3-\mathrm{D}$ case.

Eq.(10) gives explicitly the time-dependent gas distribution function $f$ at the cell boundary. Several limiting cases can be obtained, in particular: (i) in the hydrodynamic limit of $\tau<<T$ and in a smooth region one obtains schemes which are identical to these obtained from the one-step Lax-Wendroff scheme for the Navier-Stokes equations, (ii) in the limit of $\tau=\infty$ corresponding to the collisionless Boltzmann equation, our formulation reduces precisely to the kinetic representation of flux vector splitting [4] for the Euler equations, (iii) for steady state calculations, the relaxation process can be simplified by ignoring all high-order spatial and temporal terms in the expansion of $f$ and $g$ to yield

$$
f=g_{0}+e^{-t / \tau}\left(f_{0}-g_{0}\right)=g_{0}+\epsilon\left(f_{0}-g_{0}\right)
$$

The first term $g_{0}$ represents the Euler fluxes, while the second term $\epsilon\left(f_{0}-g_{0}\right)$ gives rise to a diffusion term which should be large near discontinuities to help fix a nonequi- 
librium state. Thus, $e^{-t / \tau}$ can be regarded as a diffusive parameter which should be adapted to the local flow.

It can be proved [5] that the entropy of $g_{0}$ is always larger than that of $f_{0}$, which guarantees that the local physical system will approach the state with larger entropy. This, for example, prevents the formation of unphysical rarefaction shocks.

\section{Unsteady Flow Calculations}

A forward-facing step test is carried out on a uniform mesh of $240 \times 80$ cells and the numerical results are presented in Fig.(1.a) and Fig.(1.b) for the density and entropy distribution. Here, the collision time used is equivalent to a Reynolds number of $R e \simeq 50,000$ for the upstream gas flow, taking the wind tunnel height as the characteristic length scale. A slip boundary condition is imposed in order to avoid using finer meshes close to the boundary. There is no special treatment around the corner, and we never found any expansion shocks emerging from the corner.

\section{Steady Flow Calculations with Multigrid Accel- eration}

The time independent gas-kinetic discretization scheme formulated in a previous section has been implemented in the full approximation multigrid time stepping scheme of Jameson [6]. Steady state transonic flow calculations for several airfoils have been performed to validate the simplified relaxation scheme. In these calculations, the selective parameter $\epsilon$ is determined by a switching function calculated from local pressure gradients. Using subscripts $i$ and $j$ to label the mesh cells, the switching function for fluxes in the $i$ direction is

$$
\epsilon=1-e^{-\alpha \max \left(P_{i+1, j}, P_{i, j}\right)}
$$

where $\alpha$ is a constant, and $P_{i, j}$ is an appropriate pressure switch. The computational domain is an O-mesh with 160 cells in the circumferential direction and 32 cells in the radial direction. This is a fine enough mesh to produce accurate answers with standard high resolution difference schemes. Fig. (2.a) shows the computed pressure distribution on a RAE2822 airfoil at $M_{\infty}=.75$ and 3.0 degrees angle of attack: A sharp two point shock is obtained. Fig. (2.b) shows the computed pressure distribution on a supercritical Korn airfoil at the design condition: A shock-free solution is obtained.

\section{Conclusion}

Blending the collisional gas-kinetic BGK model into the fluxes of a finite volume discretization of the conservation laws offers a promising new approach to the development of numerical hydrodynamics codes. The new scheme contains dissipation 
naturally through the kinetic flux vector splitting in the initial nonequilibrium term $f_{0}$. At the same time, the high resolution and multidimensionality of the traditional central difference schemes are recovered from the equilibrium state $g$. For steady state calculations, the coupling of a simple gas-kinetic relaxation scheme with a multigrid strategy provides fast convergence together with the favorable shock capturing properties of the gas-kinetic scheme.

\section{Acknowledgment :}

The research in this paper is supported by Grant URI/AFOSR F49620-93-1-0427.

\section{References}

[1] K.H. Prendergast and K. Xu , "Numerical Hydrodynamics from Gas-Kinetic Theory", J. of Comput. Phys. 109, 53, 1993.

[2] K. Xu , "Numerical Hydrodynamics from Gas-Kinetic Theory", Ph.D. thesis, Columbia University, 1993.

[3] A. Jameson, "Artificial Diffusion, Upwind Biasing, Limiters and their Effect on Accuracy and Multigrid Convergence in Transonic and Hypersonic Flows", AIAA paper 93-3359, 1993.

[4] J.C. Mandal andd S.M. Deshpande, "Kinetic Flux Vector Splitting for Euler Equations", Computers and Fluids, Vol.23, No.2, P447, 1994.

[5] K. Xu , L. Martinelli and A. Jameson, "Gas-Kinetic Finite Volume Methods, Flux-Vector Splitting and Artificial Diffusion", submitted to JCP, July, 1994.

[6] A. Jameson, "Transonic Flow Calculations", MAE Report \# 1651, Princeton University, March 1984. 
Density

Entropy

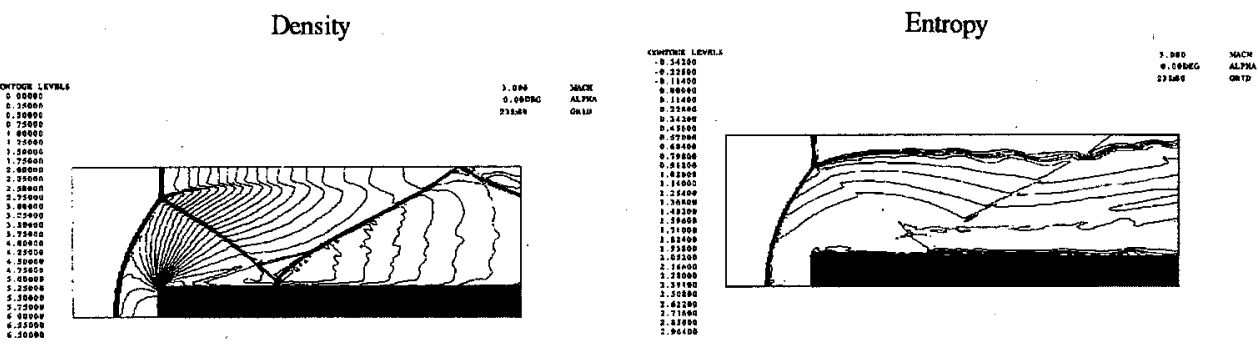

Fig. 1. Density Distribution for Mach

Fig. 1.b Entropy Distribution for Mach

3 Wind Tunnel Test on Mesh $240 \times 80$

3 Wind Tunnel Test on Mesh $240 \times 80$
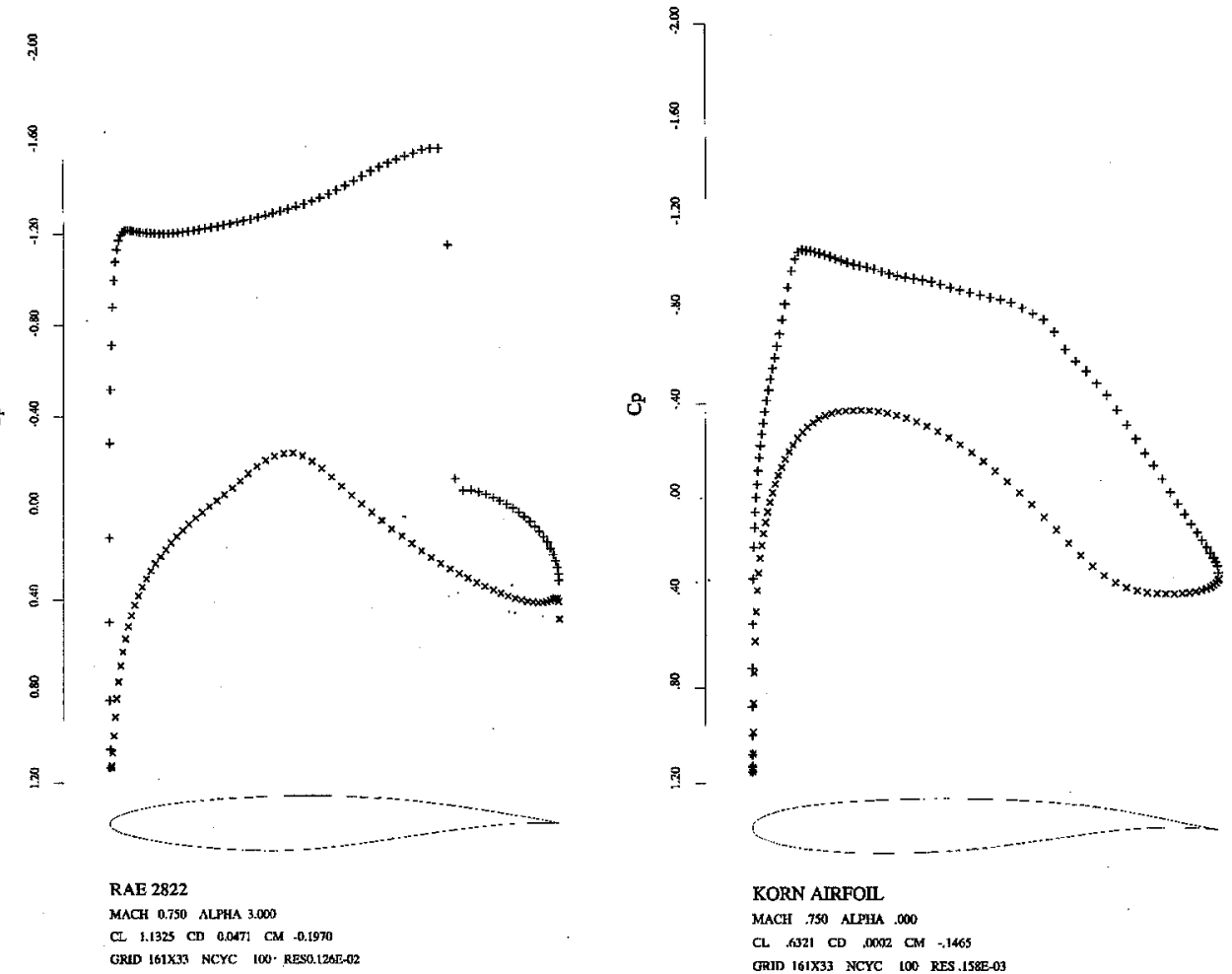

KORN AIRFOLL

MACH .750 ALPHA .000

$\begin{array}{lllllll}C L & .621 & \text { CD } & .0 \times 02 & \text { CM } & -.1465\end{array}$

GRID $161 \times 33$ NCYC LOO RES.IS8E-03

Fig. 2.a Airfoil Rae2822 with

$M=0.75, \alpha=3.0$

Fig. 2.b Korn Airfoil with $M=0.75$, $\alpha=0.0$ 